understanding as to why patients are transferred to a hospital when an onsite medical team, providing a higher level of care (HLC), is present.

Aim: To better understand the causes that necessitate patient transportation to the hospital during festivals that have onsite physician-led coverage.

Methods: De-identified patient data from a convenience sample of four, large-scale Canadian festivals (over two years) were extracted. Patient encounters that resulted in transfers to hospital, by ambulance, non-emergency transport vehicle (NETV), or self-transportation were analyzed for this study.

Results: Each festival had an onsite medical team that included physicians, nurses, and paramedics. During 34 event days, there were 10,406 patient encounters, resulting in 156 patients requiring transfer to a hospital. A patient presentation rate of $16.5 / 1,000$ was observed. The ambulance transfer rate was $0.12 / 1,000$ of attendees. The most common reason for transport was musculoskeletal injuries (54\%) that required imaging. Discussion: The presence of onsite teams capable of treating and releasing patients impacted the case mix of patients transferred to a hospital, and may reduce the number of transfers for intoxication. Confounding preconceptions, patients in the present study were transferred largely for injuries that required imaging. Results suggest that a better understanding of the specific effects onsite medical teams have on avoiding off-site transfers will aid in improving planning for music festivals. Findings also identify areas for further improvement in care, such as onsite radiology, which could potentially further reduce the impact of music festivals on local health services.

Prehosp. Disaster Med. 2019;34(Suppl. 1):s39-s40

doi:10.1017/S1049023X19000967

\section{Prediction Modeling Studies for Medical Usage Rates in Mass Gatherings: A Systematic Review Dr. Hans Van Remoortel ${ }^{1}$, Dr. Hans Scheers ${ }^{1}$, Prof. Dr. Emmy De Buck ${ }^{1,2}$, Ms. Karen Lauwers ${ }^{1}$, Prof. Dr. Philippe Vandekerckbove ${ }^{1,2}$ \\ 1. Belgian Red Cross, Mechelen, Belgium \\ 2. Department of Public Health and Primary Care, Faculty of Medicine, KU Leuven, Leuven, Belgium}

Introduction: Mass gatherings attended by large crowds are an increasingly common feature of society. In parallel, an increased number of studies have been conducted to identify those variables that are associated with increased medical usage rates.

Aim: To identify studies that developed and/or validated a statistical regression model predicting patient presentation rate (PPR) or transfer to hospital rate (TTHR) at mass gatherings. Methods: Prediction modeling studies from 6 databases were retained following systematic searching. Predictors for PPR and/or TTHR that were included in a multivariate regression model were selected for analysis. The GRADE methodology (Grades of Recommendation, Assessment, Development, and Evaluation) was used to assess the quality of evidence.

Results: We identified 11 prediction modeling studies with a combined audience of $>32$ million people in $>1500$ mass gatherings. Eight cross-sectional studies developed a prediction model in a mixed audience of (spectator) sports events, music concerts, and public exhibitions. Statistically significant variables $(\mathrm{p}<0.05)$ to predict PPR and/or TTHR were as follows: accommodation (seated, boundaries, indoor/outdoor, maximum capacity, venue access), type of event, weather conditions (humidity, dew point, heat index), crowd size, day vs night, demographic variables (age/gender), sports event distance, level of competition, free water availability, and specific TTHRpredictive factors (injury status: number of patient presentations, type of injury). The quality of the evidence was considered as low. Three studies externally validated their model against existing models. Two validation studies showed a large underestimation of the predicted patients presentations or transports to hospital (67-81\%) whereas one study overestimated these outcomes by $10-28 \%$.

Discussion: This systematic review identified a comprehensive list of relevant predictors which should be measured to develop and validate future models to predict medical usage at mass gatherings. This will further scientifically underpin more effective pre-event planning and resource provision.

Prehosp. Disaster Med. 2019;34(Suppl. 1):s40

doi:10.1017/S1049023X19000979

Proposing a Minimum Data Set for Mass Gathering Health Dr. Malinda Steenkamp ${ }^{1}$, Prof. Paul Arbon ${ }^{1}$, A/Prof. Adam Lund ${ }^{2}$, Dr. Sheila Turris' ${ }^{2}$ Dr. Jamie Ranse ${ }^{3}$, Prof. Alison Hutton ${ }^{4}$, Dr. Brendan Munn ${ }^{2}$, Dr. Ron Bowles ${ }^{5}$

1. Torrens Resilience Institute, Flinders University, Adelaide, Australia

2. University of British Columbia, Vancouver, Canada

3. Griffith University, Brisbane, Australia

4. University of Newcastle, Newcastle, Australia

5. Justice Institute of British Columbia, Vancouver, Canada

Introduction: There is currently no standardized approach to collecting mass gathering health data, which makes comparisons across or between events challenging. From 2013 onward, an international team of researchers from Australia and Canada collaborated to develop a Minimum Data Set (MDS) for Mass Gathering Health (MGH).

Aim: The process of developing the MDS has been reported on previously at the 2015 and 2017 World Congresses on Disaster and Emergency Medicine, and this presentation will present a final MDS on MGH.

Methods: This study drew from literature, including the 2015 Public Health for Mass Gatherings key considerations, previous event/patient registry development, expert input, and the results of the team's work. The authors developed an MDS framework with the aim to create an online MGH data repository. The framework was populated with an initial list of data elements using a modified Delphi technique.

Results: The MDS includes the 41 data elements in the following domains: community characteristics, event characteristics, venue characteristics, crowd characteristics, event safety considerations, public health considerations, and health services. Also included are definitions and preliminary metadata.

Discussion: The development of an MGH-MDS can grow the science underpinning this emerging field. Future input from the international community is essential to ensure that the proposed 
MDS is fit-for-purpose, i.e., systematic, comprehensive, and rigorous, while remaining fluid and relevant for various users and contexts.

Prehosp. Disaster Med. 2019;34(Suppl. 1):s40-s41

doi:10.1017/S1049023X19000980

The Sun Herald Sydney City-2-Surf Fun Run: Historical Injury Patterns and Development of a Predictive Model to Inform Health Service Planning

A/Prof. David Heslop, Mr. John Vassil, Ms. Linda Winn

UNSW Sydney, Randwick, Australia

Introduction: The Sydney City-2-Surf is the world's largest annual run entered by around 80,000 people. First aid planning at mass participation running events such as the City-2-Surf is an area in the medical literature that has received little attention. Consequently, first aid planning for these events is based on experience rather than evidence. The models for predicting casualties that currently exist in the literature are either dated or not statistically significant.

Aim: The aim of this study was to characterize patterns of injuries linked to geographic location across the course of the City2-Surf, and to explore relationships of injury types with location and meteorological conditions.

Methods: Records for formally treated casualties and meteorological conditions were obtained for the race years 2010-2016 and statistically analyzed to find associations between meteorological conditions, geographic conditions, casualty types, and location.

Results: The most common casualties encountered were heat exhaustion or hyperthermia (39.2\%), musculoskeletal (25.4\%), and physical exhaustion (10.2\%). Associations were found between gradient and the location. Type of casualty incidence with the individual distribution trends of casualty types were quite clear. Clusters of musculoskeletal casualties emerged in the parts of the course with the steepest negative gradients, while a cluster of cardiovascular events was found to occur at the top of the 'heartbreak hill,' the longest climb of the race. Regression analysis highlighted the linear relationship between the number of heat and physical exhaustion casualties and the apparent temperature (AT) at $12 \mathrm{pm}\left(\mathrm{R}^{2}=0.59, \mathrm{P}=0.044\right)$. This linear equation was used to formulate a model to predict these casualties.

Discussion: The findings of this study demonstrate the relationship between meteorological conditions, geographic conditions, and casualties. This will assist planners of other similar events to determine optimum allocation of resources to anticipated injury and illness burden.
Prehosp. Disaster Med. 2019;34(Suppl. 1):s41

doi:10.1017/S1049023X19000992

This is Sparta - A Five-Year Obstacle Course Racing Injury Analysis

Mr. Haddon Rabb ${ }^{1,3}$, Dr. Jillian Coleby ${ }^{2,3}$

1. University of British Columbia, Vancouver, Canada

2. University of Ottawa, Ottawa, Canada

3. Mass Gathering Medicine Interest Group, Vancouver, Canada

Introduction: Obstacle Course Races (OCR) are mass participation sporting events, challenging participants to complete physical and mental tasks over a variety of distances and terrains. The case series studied, Spartan Race, has races occurring in urban, rural, and wilderness venues, ranging from 5 to 42 kilometers, while incorporating 20 to 60 obstacles.

Aim: To understand the injury rates, injury and illness patterns, and transport considerations within OCRs.

Methods: A secondary data analysis of de-identified medical charts from 56 Spartan Race events occurring in Eastern Canada from 2014 to 2018 was performed. The scope of practice was first aid from 2014 to 2017, with the addition of advanced life support onsite in 2018.

Results: Over 5 years, 2,387 injuries occurred among 127,481 participants, creating a patient presentation rate of 18.7/1000. Although the majority of injuries $(92 \% ; n=2,204)$ were treated onsite, a transport to hospital rate of $1.2 / 1000(n=154)$ occurred along with an ambulance transport rate of $0.23 / 1000(n=29)$. Lacerations (55\%) and musculoskeletal (36\%) injuries were the most frequent clinical presentations observed, whereas lifethreatening emergencies (affecting airway, breathing, and circulation) were infrequent $(\mathrm{n}=10)$. Transport to the closest local tertiary care center was on average 49.8 kilometers ( 25.3 kilometers) and 40.5 minutes (17.9 minutes) away from the venue.

Discussion: These results suggest that there may be an upper limit to the injury rates within Spartan Races. The majority of patient presentations were able to be treated onsite, supporting the need for a qualified onsite medical team to mitigate the strain on local healthcare systems. Although life-threatening emergencies were uncommon, they do occur, and medical teams must be appropriately prepared. Further research is needed to understand the staffing and equipment requirements of medical teams, the demographic information of the injured, and the examination of the impact OCR events have on the local health care systems.

Prehosp. Disaster Med. 2019;34(Suppl. 1):s41

doi:10.1017/S1049023X19001006 